\title{
Análise de detecção de cluster na caracterização espaço- temporal da tuberculose bovina no Estado da Bahia ${ }^{1}$
}

\author{
Luciana N. Ávila ${ }^{2 *}$, Andrés M. Perez ${ }^{3}$, José S. Ferreira Neto ${ }^{4}$ Fernando Ferreira ${ }^{4}$, \\ Evelise 0. Telles ${ }^{4}$, Ricardo A. Dias ${ }^{4}$, Marcos Amaku$^{4}$ e Vitor S.P. Gonçalves ${ }^{5}$
}

\begin{abstract}
L.A., Perez A.M., Ferreira Neto S.J., Ferreira F., Telles O.E., Dias A.R., Amaku M. \& Gonçalves V.S.P. 2009. [Cluster detection analyses for temporal-spatial characterization of bovine tuberculosis in Bahia, Brazil.] Análise de detecção de cluster na caracterização espaço-temporal da tuberculose bovina no Estado da Bahia. Pesquisa Veterinária Brasileira 33(11):1313-1318. Programa Nacional de Controle e Erradicação da Brucelose e Tuberculose Bovina, Agência Estadual de Defesa Agropecuária da Bahia, Secretaria de Agricultura do Estado da Bahia, Av. Ademar de Barros 967, Salvador, BA 40170-110, Brazil. E-mail: luciana.avila@adab.ba.gov.br

Bovine tuberculosis (BTB) is a disease caused by the infection with Mycobacterium bovis that affects humans and several mammalian species. BTB is important, because it inflicts farreaching economic losses to infected regions, and due to its impact on public health. Epidemiological surveys were conducted in the State of Bahia between 2008 and 2010 with the objective to estimate the prevalence and to assess the spatial distribution of the disease. The State of Bahia has been stratified into four regions, each of them representing a set of homogeneous epidemiological and demographic characteristics, referred to as Production Circuits. A total of 18,810 more than 2-year-old cattle in 1,305 herds, ranging from 320 to 370 ones per region, and 20 to 40 cattle per herd were randomly selected. A cervical comparative test was applied to each selected animal; reactive cattle and cattle with two consecutive inconclusive tests were considered BTB-positive, whereas non-reactive cattle were considered BTBnegative. Positive herds were classified as those with $\geq 20$ sampled cattle and at least one BTB-positive, as well as those with 40 cattle sampled with $\geq 2$ BTB-positive animals. Latitude and longitude were recorded for each sampled herd with a generic Global Positioning System (GPS). The Cuzick-and-Edwards' test and the spatial scan statistic were used to assess whether BTB was spatially clustered. Herd-level prevalence, as indicated by the proportion of case-herds, was $1.6 \%$ (range 0.3 to $2.9 \%$ per region). No significant evidence $(\mathrm{P}<0.05)$ of spatial clustering was detected, most likely due to the low disease prevalence in the region. Results here suggest that BTB is low prevalent in the State of Bahia and that under these conditions epidemiological outbreaks found cannot be explained by spatially-structured factors.
\end{abstract}

INDEX TERMS: Diseases of cattle, infectious diseases, bovine tuberculosis, temporal-spatial analysis.

RESUMO.- A tuberculose bovina (BTB) é uma enfermidade causada pela infecção pelo Mycobacterium bovis que acomete o homem e diversas espécies de mamíferos. A BTB

\footnotetext{
${ }^{1}$ Recebido em 13 de julho de 2013.

Aceito para publicação em 1 de outubro de 2013.

${ }^{2}$ Programa Nacional de Controle e Erradicação da Brucelose e Tuberculose Bovina (PNCEBT), Agência Estadual de Defesa Agropecuária da Bahia (ADAB), Secretaria de Agricultura do Estado da Bahia, Av. Ademar de Barros 967, Salvador, BA 40170-110, Brasil. *Autor para correspondência: luciana.avila@adab.ba.gov.br

${ }^{3}$ Center for Animal Disease Modeling and Surveillance, VM: Medi-
}

tem grande importância por causar prejuízos econômicos nas regiões infectadas e por seu impacto na saúde pública. Foi realizado inquérito epidemiológico no Estado da Bahia,

cine and Epidemiology, UC Davis, One Shields Avenue, Davis, CA 95616, USA.

${ }^{4}$ Departamento de Medicina Veterinária Preventiva e Saúde Animal, Universidade de São Paulo (USP), Av. Prof. Orlando Marques de Paiva 87, São Paulo, SP 05508-270, Brasil.

${ }^{5}$ Faculdade de Agronomia e Veterinária, (FAV) Universidade de Brasília (UnB), Campus Darcy Ribeiro, Asa Norte, ICC Sul 4508, Brasília, DF 70910970, Brasil. 
entre 2008 e 2010, com o objetivo de estimar a prevalência e conhecer a distribuição espaço temporal da enfermidade. 0 Estado foi estratificado em quatro regiões, cada uma com características epidemiológicas e demográficas homogêneas representativas de formas de produção pecuária. Um total de 18.810 cabeças com idade superior a 2 anos foi amostrado em 1350 propriedades. 0 teste cervical comparativo foi aplicado em cada animal selecionado, sendo considerados positivos os animais reagentes positivos ou duas vezes inconclusivos. Latitude e Longitude foram tomadas para cada propriedade amostrada com o auxilio do aparelho de Global Positioning System (GPS). 0 teste de Cuzick-and-Edwards e a análise de rastreio espacial (spatial scan statistic) foram utilizados para identificar qualquer agrupamento espacial de BTB. A prevalência de rebanho na Bahia, indicando a proporção de propriedades foco, foi de 1,6\% (IC 95\%: 1,0\% - 2,69\% por região). Nenhuma evidência significativa $(\mathrm{P}<0.05)$ de aglomeração espacial ou clustering foi detectada, possivelmente devido à baixa prevalência da doença. Estes resultados sugerem que a BTB tem baixa prevalência no estado da Bahia e que, nestas condições epidemiológicas, os focos encontrados não podem ser explicados por fatores espacialmente estruturados.

TERMOS DE INDEXAÇÃO: Doenças de bovinos, doenças infecciosas, tuberculose bovina, analise espaço-temporal.

\section{INTRODUÇÃO}

A tuberculose bovina (BTB) é uma enfermidade infecto-contagiosa, que afeta os animais de produção e o homem e se caracteriza por ter uma evolução crônica, afetando principalmente o sistema respiratório. A doença tem distribuição mundial (Campos 2008), sendo sua prevalência mais alta nos paises em desenvolvimento e em rebanhos leiteiros, principalmente naqueles com certo grau de tecnologia de produção (Roxo 1997).

No Brasil, o Programa Nacional de Controle e Erradicação da Brucelose e Tuberculose Animal (PNCEBT) tem como objetivo combater estas enfermidades, reduzir sua incidência e prevalência, a fim de minimizar os prejuízos econômicos causados e garantir a segurança alimentar da carne e produtos lácteos ofertados ao consumidor (Brasil 2006). O programa tem características similares aos programas de controle e erradicação da tuberculose bovina desenvolvidos em vários outros países, especialmente nas Américas, que se baseiam no uso da prova cutânea de tu- berculina para diagnostico da tuberculose e o sacrifício dos animais reagentes. Ademais se preconiza o controle do movimento dos animais, a formação e capacitação de veterinários para o diagnóstico da tuberculose bovina e a certificação de propriedades livres ou monitoradas para a tuberculose e brucelose (Brasil 2004).

A tuberculose bovina está presente em todo o território brasileiro, porém, a sua prevalência e distribuição regional ainda não foram bem caracterizadas. Notificações oficiais existentes no Brasil, nos últimos dez anos indicaram uma prevalência bastante heterogênea. Belchior (2000) encontrou em Minas Gerais uma prevalência de 5\% de rebanhos positivos e $0,8 \%$ de animais positivos e concluiu que os fatores de risco para a tuberculose bovina encontrados nesse estudo - sistema de produção, grupo genético, sistema de ordenha, resfriamento do leite e monitoramento da produção - estão relacionados à intensificação da produção. Silva (2012) encontrou uma prevalência de 2,15\% de rebanhos positivos e $0,42 \%$ de animais positivos no Estado do Paraná e Nespoli (2012) encontrou para o Estado do Mato Grosso, uma prevalência de rebanhos e de animais positivos de $1,3 \%$ e $0,123 \%$ respectivamente. A heterogeneidade dos valores de prevalência da tuberculose nas diferentes regiões pode estar relacionada com vários fatores tais como fonte de aquisição dos animais, clima, serviços de diagnóstico da tuberculose em cada propriedade (Poletto et al. 2004, Oliveira 2008), sistema de produção, tamanho do rebanho, idade, raça, grau de tecnificação no modo de produção da propriedade e presença de animais para produção de carne e de leite no mesmo rebanho (Perez et al. 2002, Flores et al. 2005, Elias et al. 2008, Nespoli 2012).

No estado da Bahia, foi realizado um estudo para caracterizar a situação epidemiológica da doença no ano de 2008 a 2010. Foram amostrados 18.810 animais, em 1.305 propriedades. As prevalências encontradas de focos e de animais (fêmeas e machos com idade maior ou igual a 24 meses) foram respectivamente de 1,6\% [IC 95\%: 1,0-2,6\%] e $0,21 \%$ [IC 95\%: 0,07-0,6\%], conforme pode ser consultado no Quadro 1 (Costa 2012). Em virtude das baixas prevalências encontradas para a BTB no estado da Bahia, os esforços atuais do programa têm se focado na adoção de medidas visando a identificação geográfica de focos, para rastreamento e saneamento dos mesmos. Assim, torna-se necessário usar técnicas que possibilitem conhecer a com-

Quadro 1. Dados censitários e de prevalência aparente de focos de tuberculose bovina nos circuitos produtores e no Estado da Bahia

\begin{tabular}{|c|c|c|c|c|c|c|}
\hline $\begin{array}{l}\text { Circuitos } \\
\text { pecuários }\end{array}$ & $\begin{array}{l}\text { Número de } \\
\text { municípios }\end{array}$ & $\begin{array}{c}\text { Total de } \\
\text { propriedades } \\
\text { com atividade } \\
\text { reprodutiva }\end{array}$ & $\begin{array}{c}\text { Propriedades } \\
\text { testadas }\end{array}$ & $\begin{array}{l}\text { Propriedades } \\
\text { Positivas }\end{array}$ & $\begin{array}{c}\text { Prevalência } \\
\text { (\%) }\end{array}$ & IC 95\% (\%) \\
\hline 1 & 132 & 60.272 & 344 & 7 & 2,0 & $(1,0-2,0)$ \\
\hline 2 & 99 & 91.929 & 308 & 9 & 2,9 & $(1,5-5,5)$ \\
\hline 3 & 124 & 85.448 & 335 & 1 & 0,3 & $(0,04-2,1)$ \\
\hline 4 & 62 & 31.384 & 318 & 2 & 0,6 & $(0,2-2,5)$ \\
\hline Total & 417 & 269.033 & 1.305 & 19 & 1,6 & $(1,00-2,6)$ \\
\hline
\end{tabular}

Fonte: Agência Estadual de Defesa Agropecuária da Bahia, 2008. IC = Intervalo de confiança. Efeito do desenho. 
plexidade envolvida na distribuição espaço-temporal da doença e a possivel presença de áreas de maior ocorrência da mesma, recorrendo a sistemas de informação geográfica e análises espaciais, permitindo otimizar investimentos em seu controle.

0 tratamento da informação geográfica vem influenciando de maneira crescentes várias áreas do conhecimento. É importante questionar se os dados referentes aos fenômenos pesquisados estão distribuídos espacialmente de forma aleatória ou se estão aglomerados em áreas específicas. Como define o Código Sanitário para os Animais Terrestres da Oficina Internacional de Epizootias (OIE 2012), um dos objetivos principais da vigilância epidemiológica é identificar grupos ou "clusters" de indivíduos ou populações em risco de introdução ou disseminação de enfermidades animais e assim conhecer sua distribuição temporal e/ou espacial. Neste trabalho foi adotado o uso do termo inglês "cluster" para representar "conglomerado", pois o seu uso já está bastante difundido. Segundo Tavares (2009), um cluster espacial é parte de um mapa em que a ocorrência de casos de um fenômeno de interesse é discrepante do restante do mapa (alta demais ou baixa demais); neste estudo o fenômeno está representado pela infecção por BTB.

O objetivo do presente estudo foi identificar possíveis áreas de maior risco de ocorrência de BTB no estado da Bahia, com base nos dados gerados pelo estudo de prevalência realizado entre 2008 e 2012, e discutir aspectos relacionados com as metodologias espaciais utilizadas. Os resultados ajudarão a entender as aplicações de técnicas para detecção de clusters ou agrupamento espacial de tuberculose e outras enfermidades infecciosas na Bahia, assim como em outras regiões do Brasil.

\section{MATERIAL E MÉTODOS}

\section{Origem dos dados}

O rebanho bovino baiano está estimado, segundo ultimo levantamento da campanha de vacinação contra febre aftosa 20112, realizada pela Agência de Defesa Agropecuária do Estado da Bahia (ADAB), em mais de 11.000.000 de cabeças distribuídas em cerca de 278 mil propriedades ou rebanhos (Bahia, 2012). A Bahia ocupa a nona posição no rebanho bovino do país, o que demonstra a importância dessa espécie para a economia agropecuária como um todo (IBGE 2010).

Para este estudo foram utilizados dados geográficos gerados pelo inquérito da BTB realizado para o estado da Bahia no período de outubro de 2008 a novembro de 2010. Este inquérito foi planejado em colaboração com o Ministério da Agricultura Pecuária e Abastecimento (Mapa), a ADAB, a Faculdade de Medicina Veterinária e Zootecnia da Universidade de São Paulo (USP) e a Universidade de Brasília (UnB). A Bahia foi dividida em quatro regiões com características homogêneas, denominadas neste estudo de Circuitos Pecuários (Fig.1). Cada região foi definida empiricamente com base nas características de produção e comercialização pecuária visando a observação das variações regionais destes parâmetros com a epidemiologia da tuberculose bovina. Foram utilizados como critérios na definição dos circuitos pecuários: as tipologias de produção pecuária, práticas de manejo, finalidades de exploração, tamanho médio de rebanhos e sistemas de comercialização. A divisão também levou em consideração a capacidade operacional da $\mathrm{ADAB}$, já que o incremento do número

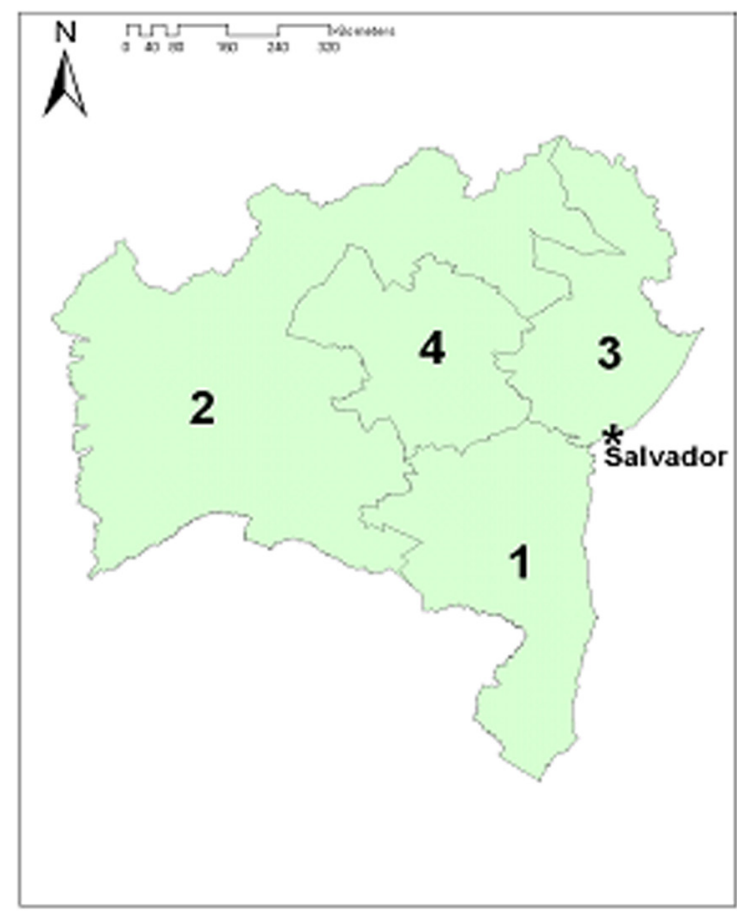

Fig.1. Mapa do Estado da Bahia evidenciando os quatro circuitos pecuários, 2008.

de regiões amostrais levaria ao aumento do número de propriedades a serem amostradas.

Em cada circuito foram amostradas pelo menos 300 propriedades dedicadas à pecuária bovina, com atividade reprodutiva. No total foram amostrados 1.305 rebanhos (propriedades) e 18.810 animais (bovinos com idade superior a 2 anos), conforme pode ser consultado no Quadro 1. O diagnóstico foi realizado de acordo com o protocolo de diagnóstico para tuberculinização comparada ou Teste Cervical Comparativo (TCC) e a leitura realizada por médicos veterinários do serviço oficial. Os animais que resultaram inconclusivos foram retestados com o mesmo procedimento diagnóstico em intervalo mínimo de 60 dias. Para cada propriedade sorteada aleatoriamente foram amostrados animais de maneira aleatória e sistemática, respeitando o seguinte critério: rebanhos com até 99 bovinos (machos e fêmeas com idade $\geq 24$ meses) foram amostrados 20 animais com idade $\geq 24$ meses ou todos os animais desta faixa etária quando eles eram em quantidade inferior a 20. Em propriedades com $\geq 100$ bovinos (machos e fêmeas com idade $\geq 24$ meses) foram amostrados 40 animais dentro desta faixa etária. Propriedades com até 20 bovinos amostrados foram consideradas foco quando pelo menos um animal foi positivo. Propriedades com 40 bovinos amostrados foram consideradas foco quando dois ou mais animais foram positivos (Costa 2012). 0 método de amostragem e os critérios de classificação de focos aplicados visaram garantir boa sensibilidade e especificidade de rebanho (agregada) para minimizar a proporção de propriedades falso-negativas e falso-positivas, respectivamente.

\section{Análise dos dados}

Uma vez identificados os focos de BTB, cada propriedade amostrada teve sua identificação geográfica individual (georreferenciadas com o auxilio do aparelho de Global Positioning System - GPS) e foi classificada como propriedade foco ou caso (1) ou propriedade controle (0). Assim, a agregação de dados para casos e para controles foi definida pela distribuição real em cada uma das quatro regiões amostrais no estado da Bahia, e obtida 


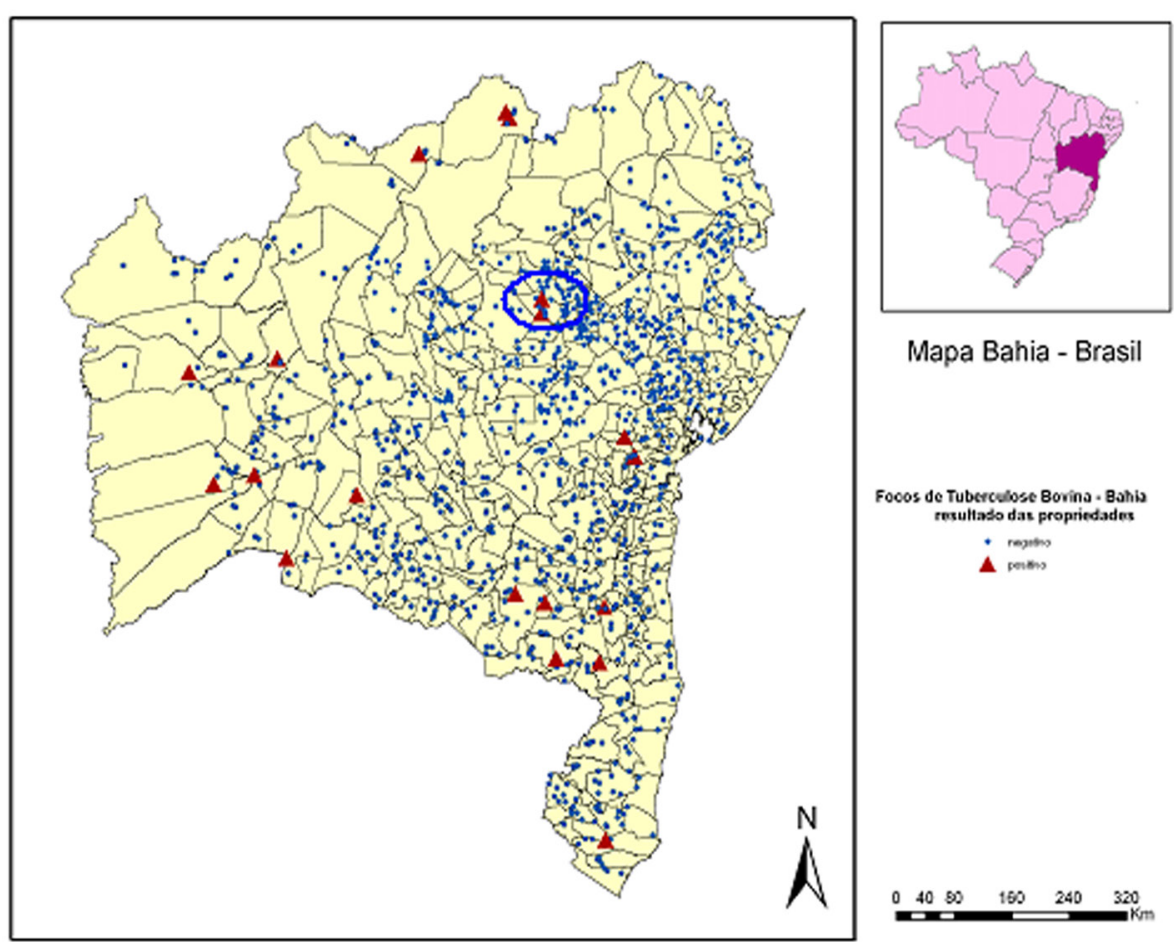

Fig.2. Distribuição espacial dos focos de tuberculose bovina no estado da Bahia, evidenciando a falsa impressão de existência de um cluster no município de Miguel Calmon, 2008.

por meio de uma amostragem aleatoria simples. Os resultados do teste diagnóstico para BTB foram então cruzados com as coordenadas geográficas (latitude e longitude) para realização da analise espacial. Todas estas informações coletadas foram utilizadas para identificar áreas geográficas de maior risco para a BTB na Bahia.

Estas áreas foram identificadas utilizando técnicas para identificação de agrupamentos do tipo rastreio espacial (spatial scan statistic) (Kulldorff \& Nagarwalla 1995) e teste Cuzick and Edwards (Cuzick \& Edwards 1990). Segundo Ward \& Carpenter (2000) é recomendado que se utilize ambas as técnicas combinadas, quando se pretende identificar agrupamentos de doenças em populações animais. Estas técnicas são complementares; o teste Cuzick and Edwards informa se um agrupamento está ou não presente, enquanto a análise de agrupamentos do tipo rastreio espacial permite identificar sua localização (primária e/ou secundaria) onde é mais provável que a doença esteja agrupada.

Neste estudo, a técnica de clustering espaço-temporal foi aplicada duas vezes; primeiramente aplicada ao estado da Bahia como um todo e posteriormente em separado (para cada circuito produtor) a fim de se avaliar os possíveis efeitos de quaisquer agrupamentos encontrados. Esta técnica se baseia na comparação de parâmetros das distribuições probabilísticas de sucessivas janelas espaço-temporais de varrimento, com os parâmetros das distribuições das áreas vizinhas, procurando evidência estatística da sua diferença e podendo assumir diversas distribuições $(\mathrm{Nu}-$ nes et al. 2007).

Foi adotada na spatial scan statistic a distribuição do tipo Bernoulli (Kulldorff \& Nagarwalla 1995), no qual os casos e não-casos são representados por uma variavel $0 / 1$, correspondendo a focos e não focos. As propriedades focos e não focos foram classificadas como casos e controles e a análise adotada foi puramente espacial. O software utilizado foi o SATScan versão 9.1.1 e para o teste Cuzick and Edwards o software utilizado foi o ClusterSeer. Posteriormente foram construidos mapas para representar os focos de BTB no estado com auxilio do programa ArcMap 9.1 (ESRI $\left.{ }^{\odot} 2005\right)$.

\section{RESULTADOS}

Não foram identificadas áreas de maior risco de ocorrência de BTB no estado da Bahia, uma vez que não houve evidencia significativa $(\mathrm{P}<0.05)$ de agrupação espacial detectada pelo teste de Cuzick-and-Edward em qualquer dos níveis mais próximos de vizinhança investigados, que variou de 1 a 10, sendo a ordem de proximidade de vizinhança 1 a primeira ordem, ou o vizinho mais próximo.

$\mathrm{Na}$ análise do tipo spatial scan statistic não foi encontrada presença de cluster para BTB quando realizada para o estado da Bahia como um todo, sem considerar a divisão em circuitos produtores. Todavia as análises espaciais em separado para cada circuito produtor, sugeriram haver um "cluster" $(p<0,028)$ no circuito produtor quatro, especificamente no município de Miguel Calmon, Bahia (Fig.2).

\section{DISCUSSÃO}

A ausência de agrupamentos espaciais de focos de tuberculose bovina na Bahia pode estar relacionada à baixa prevalência da doença no Estado. É possível concluir que os focos encontrados não podem ser explicados por fatores espacialmente estruturados.

Observou-se ainda que as complexidades envolvidas na aplicação da metodologia de análise de cluster espacial e na interpretação de seus resultados podem conduzir a conclusões errôneas, uma vez que se esta análise houvesse sido aplicada para cada circuito pecuário do Estado da Bahia em separado, poderia levar a falsa impressão de existência de um cluster. É provável que o cluster de Miguel Calmon seja na realidade produzido pelo efeito de ambas as propriedades foco incluídas no cluster estarem 
localizadas no limite (ou borda) do circuito produtor (ou da regional) analisado, podendo levar a erro de identificação de cluster. Na verdade se trata de uma divisão geográfica artificial (empírica) criada neste estudo para fins de análise e que logicamente não está sujeita aos parâmetros reais de ocorrência das enfermidades, que não respeitam limites geográficos. Este efeito denominado "efeito de borda" se dá quando analisamos somente cada circuito produtor em separado, havendo a impressão de haver um cluster. Quando agregamos informações referentes às demais propriedades do circuito produtor vizinho, incluímos as informações de propriedades negativas que integram a janela ou raio de análise (neste estudo pode ser definido no raio de $11 \mathrm{~km}$ ), desaparecendo então o cluster espacial.

A detecção de agrupamento espaço-temporal tem sua complexidade e limitações, contudo, o ganho com a obtenção de resultados de maior precisão e segurança para tomada de decisões conduz a maior eficiência das ações de defesa sanitaria. As limitações dessa metodologia envolvem: a necessidade de conhecimento por parte do investigador das características dos processos estocásticos em causa, a sensibilidade do método à variabilidade decorridas do grau de subdetecção quando variam no espaço ou no tempo ou as variações impostas por limites artificiais na definição de áreas de estudos. Segundo Buehler et al. (2004) a importância de utilizar essa metodologia está em que na maioria dos casos uma aparente homogeneidade (ou heterogeneidade) de uma determinada distribuição, numa representação gráfica clássica, pode esconder uma distribuição heterogênea (ou homogênea), que a análise estatística de clustering vem a revelar. Reforça-se, portanto, a necessidade de sempre se aplicar uma abordagem epidemiológica crítica, respeitando-se o contexto dos demais conhecimentos envolvidos. Kulldorf (1997) \& Nunes et al. (2007) alertam ainda para a necessidade de se conhecer adequadamente a natureza e a história natural do fenômeno em análise.

\section{CONCLUSÃO}

Podemos concluir que os ganhos em qualidade dos resultados aportados e a vasta aplicabilidade da metodologia na área de epidemiologia e defesa sanitária são animadores e representam um considerável ganho à interpretação epidemiológica da distribuição espaço-temporal de enfermidades e, consequentemente, à qualidade das decisões baseadas nessa evidência.

Agradecimentos.- Este projeto representa parte da dissertação de doutorado submetida pela autora para a Universidade de Buenos Aires, Argentina. Para tal foram utilizados dados obtidos através do inquérito epidemiológico da tuberculose bovina realizado no estado da Bahia, planejado em colaboração com o Ministério da Agricultura Pecuária e Abastecimento (Mapa), a Agência de Defesa Agropecuária do Estado da Bahia (ADAB), Centro Colaborador em Saúde Animal da Faculdade de Medicina Veterinária e Zootecnia da Universidade de São Paulo (USP) e o Laboratório de Epidemiologia Veterinária da Universidade de Brasília (UnB). As análises estatísticas dos dados foram realizadas em parceria com o Center for Animal Disease Modeling and Surveillance (UC Davis) e a UnB, lotação profissional dos supervisores e co-supervisores da orientada.

\section{REFERÊNCIAS}

Abrahão R.M.C.M. 1999. Tuberculose humana causada pelo Mycobacterium bovis: considerações gerais e a importância dos reservatórios animais. Archs Vet. Sci. 4:5-15.

ADAB 2012. Relatório semestral da campanha de erradicação da Febre Aftosa. Agência Estadual de Defesa Agropecuária da Bahia, Salvador, BA.

Amorim B.M. 2008. Prevalência da tuberculose em bovinos diagnosticados pelo teste de tuberculinização nas regiões do estado do Espírito Santo, Brasil. Monografia de Pós-Graduação em Defesa e Vigilância Sanitária Animal, Instituto Brasileiro de Pósgraduação Qualittas, Universidade Castelo Branco, Vitória, ES. 76p.

Arcelles M. 2005. Prevalência de tuberculosis bovina em el distrito de Végueta, Huaurá, Peru. Revta Invest. Vet. Peru 16:154-157.

Belchior A.P.C. 2000. Prevalência, distribuição regional e fatores de risco da tuberculose bovina em Minas Gerais. Dissertação de Mestrado em Medicina Veterinária Preventiva e Epidemiologia, Escola de Veterinária, Universidade Federal de Minas Gerais, Belo Horizonte. 55p.

Brasil 2001. Programa Nacional de Controle e Erradicação da Brucelose e da Tuberculose Animal (PNCEBT). Ministério da Agricultura, Pecuária e Abastecimento, Brasília.

Brasil 2006. Manual Técnico do Programa Nacional de Controle e Erradicação da Brucelose e da Tuberculose Animal. Ministério da Agricultura, Pecuária e Abastecimento, Brasília.

Buehler J.W., Hopkins R.S., Overhage J.M., Sosin D.M. \& Van Tong. 2004. Framework for evaluating public health surveillance systems for early detection of outbreaks. CDC 53:1-11.

Campos S.S. 2008. Prevalência da tuberculose bovina. Revta Eletrônica do CESVA 1:245.

Costa L. 2012. Dissertação de Mestrado em Ciência animal nos trópicos, Escola de medicina veterinária e zootecnia, Universidade Federal da Bahia, Salvador, BA. 121p.

Cosivi O., Grange J.M., Daborn C.J., Ravigilone M.C., Fujikura T., Cousins D., Robinson R.A., Huchzermeyer H.F.A.K., De Kanntor I. \& Meslin F.X. 1998. Zoonotic tuberculosis due to Mycobacterium bovis in developing countries. Emerg. Infect. Dis. 4:59-70.

Cuzick J. \& Edwards R. 1990. Spatial clustering for inhomogeneous populations. J. Royal Statistical Society B 52:73-104.

Elias K., Hussein D., Asseged B., Wondwossen T. \& Gebeyehu M. 2008. Status of bovine tuberculosis in Addis Ababa dairy farms. Revta Scient. Technol. 27:915-923.

Elliott P. \& Wartenberg D. 2004. Spatial epidemiology: current approaches and future challenges. Environ. Health Perspectives 112:998-1006.

Flores L.L., Pai M., Colford J.M. \& Riley L.W. 2005. In-house acid amplification test for the detection of Mycobacterium tuberculosis in sputum specimens: meta-analysis and metaregression. BMC Microbiol. 5:55.

Gil A.D. \& Samartino L. 2000. Zoonosis en los sistemas de producción animal de las áreas urbanas y periurbanas de América Latina: Livestock Policy Discusión Paper nº 2. Livestock Information and Policy Branch, Food and Agriculture Organization (AGAL-FAO), Rome, p.16-22.

Griffin J.M., Martins S.W. \& Thorburn M.A. 1996. A case-control study on the association of selected risk factors with the occurrence of bovine tuberculosis in the Republic of Ireland. Prev. Vet. Med. 34:217-229.

IBGE 2010. Pesquisa Pecuária Municipal. Instituto Brasileiro de Geografia e Estatística, Brasília, DF.

Kulldorff M. 1997. A spatial scan statistic. Communications in Statistics, Theory and Methods 26:1481-1496.

Kulldorff M. \& Nagarwalla N. 1995. Spatial disease clusters: detection and inference. Statistics in Medicine 14:799-810.

Mangoni J. 2009. Ocorrência de tuberculose e de brucelose em rebanhos da região Sudoeste do Paraná. Revta Cient. Eletrôn. Med. Vet. 12:1-9.

Martínez-López B., Perez A.M. \& Sánchez-Vizcaíno J.M. 2009. Combined application of social network and cluster detection analyses for temporal-spatial characterization of animal movements in Salamanca, Spain. Prev. Vet. Med. 91:29-38. 
Moran P.A.F. 1950. Notes on continous stochastic phenomena. Biometrika, London, 37:17-23.

Nespoli J.M.B. 2012. Situação epidemiológica da tuberculose bovina no Estado de Mato Grosso. Dissertação de Mestrado, Faculdade de Medicina Veterinária e Zootecnia, Universidade de São Paulo, São Paulo, SP. Recuperado em 2013-07-09, de http://www.teses.usp.br/teses/disponiveis/10/10134/tde-28092012-165944/

Nunes C., Briz T., Gomes D. \& Dias C.M. 2007. Tuberculosis incidence in Portugal: spatiotemporal clustering. Revta Port. Saúde Publica 26:514p.

Perez A.M., Ward M.P., Torres P. \& Ritacco V. 2002. Use of spatial and monitoring data to identify clustering of bovine tuberculosis in Argentina. Prev. Vet. Med. 56:63-74.

Perez A.M., Thurmond M.C., Grant P.W. \& Carpenter T.E. 2005. Use of the scan statistic on disaggregated province-based data: Foot-and-mouth disease in Iran. Prev. Vet. Med. 71:197-207.

Silva M.C.P. 2012. Epidemiologia e fatores de risco da tuberculose bovina no Paraná. Tese de Doutorado em Ciência Animal, Centro de Ciências Agrarias, Universidade Estadual de Londrina, Londrina, PR. 82p.

Ribeiro A.R.P., Lobato F.C.F. \& Abreu V.L.V. 2003. Prevalência de tuberculose e brucelose bovina no município de Ilhéus. Arq. Bras. Med. Vet. Zootec. 55:120-122.
Roxo E. 1997. Mycobacterium bovis como causa de zoonose. Revta Ciênc. Farmacêut. 18:101-108.

Ruggiero A.P., Ikuno A.A., Ferreira V.C.A. \& Roxo E. 2007. Tuberculose bovina: alternativas para o diagnóstico. Arqs Inst. Biológico, São Paulo, 74: 55-65.

Tavares R. 2009. Extensões da Estatística Scan Espacial utilizando Técnicas de Otimização Multiobjetivo. Tese de Doutorado em Estatística, Departamento de Estatística, Universidade Federal de Minas Gerais, Belo Horizonte. $127 \mathrm{p}$.

Oliveira A.P. 2008. Caracterização epidemiológica dos pacientes que abandonaram o tratamento de tuberculose em Maceió, AL. Revta Bras. Pneumol. Sanit. 16:123-128.

OIE 2009. Bovine Tuberculosis. OIE Terrestrial Manual, Paris.16p.

Poletto R., Kreutz L.C., Gonzales J.C. \& Barcellos L.J.G. 2004. Prevalência de tuberculose, brucelose e infecções víricas em bovinos leiteiros do município de Passo Fundo, RS. Ciência Rural 34:595-598.

Ward M.P. \& Carpenter T.E. 2000a. Analysis of time-space clustering in veterinary epidemiology. Prev. Vet. Med. 43:225-237.

Ward M.P. \& Carpenter T.E. 2000b. Techniques for analysis of disease clustering in space and in time in veterinary epidemiology. Prev. Vet. Med. $45: 257-284$ 